\title{
Chronic Stress Increases the Plasmalemmal Distribution of the Norepinephrine Transporter and the Coexpression of Tyrosine Hydroxylase in Norepinephrine Axons in the Prefrontal Cortex
}

\author{
LeeAnn H. Miner, ${ }^{1}$ Hank P. Jedema, ${ }^{1}$ Forrest W. Moore, ${ }^{1}$ Randy D. Blakely, ${ }^{3}$ Anthony A. Grace, $, 1,2$ and Susan R. Sesack ${ }^{1}$ \\ ${ }^{1}$ Departments of Neuroscience and Psychiatry and ${ }^{2}$ Department of Psychology, University of Pittsburgh, Pittsburgh, Pennsylvania 15260, and ${ }^{3}$ Department \\ of Pharmacology, School of Medicine, and Center for Molecular Neuroscience, Vanderbilt University, Nashville, Tennessee 37232
}

\begin{abstract}
Norepinephrine (NE) potently modulates the cognitive and affective functions of the prefrontal cortex (PFC). Deficits in NE transmission are implicated in psychiatric disorders, and antidepressant drugs that block the NE transporter (NET) effectively treat these conditions. Our initial ultrastructural studies of the rat PFC revealed that most NE axons (85-90\%) express NET primarily within the cytoplasm and lack detectable levels of the synthetic enzyme tyrosine hydroxylase (TH). In contrast, the remaining $10-15 \%$ of PFC NE axons exhibit predominantly plasmalemmal NET and evident TH immunoreactivity. These unusual characteristics suggest that most PFC NE axons have an unrecognized, latent capacity to enhance the synthesis and recovery of transmitter. In the present study, we used dual-labeling immunocytochemistry and electron microscopy to examine whether chronic cold stress, a paradigm that persistently increases NE activity, would trigger cellular changes consistent with this hypothesis. After chronic stress, neither the number of profiles exhibiting NET labeling nor their size was changed. However, the proportion of plasmalemmal NET nearly doubled from $29 \%$ in control animals to $51 \%$ in stressed rats. Moreover, the expression of detectable TH in NET-labeled axons increased from only $13 \%$ of profiles in control rats to $32 \%$ of profiles in stressed animals. Despite the consistency of these findings, the magnitude of the changes varied across individual rats. These data represent the first demonstration of activity-dependent trafficking of NET and expression of TH under physiological conditions and have important implications for understanding the pathophysiology and treatment of stress-related affective disorders.
\end{abstract}

Key words: catecholamine; trafficking; post-traumatic stress disorder; depression; antidepressants; attention deficit hyperactivity disorder

\section{Introduction}

Norepinephrine (NE) projections to the prefrontal cortex (PFC) modulate crucial cognitive functions, including attention and arousal (Robbins, 1984; Aston-Jones et al., 1999). Moreover, NE mediates many of the adaptive/maladaptive consequences of stress exposure (Stanford, 1995; Zigmond et al., 1995), implicating this system in the pathophysiology of depression, posttraumatic stress disorder, and attention deficit hyperactivity disorder (ADHD) (Callado et al., 1998; Biederman and Spencer, 1999; Southwick et al., 1999). The temporal and spatial dynamics of NE transmission are potently regulated by reuptake through the NE plasma membrane transporter (NET) (Blakely and Bauman, 2000), and drugs blocking NET effectively treat mood dis-

Received 0ct. 18, 2005; revised Dec. 7, 2005; accepted Dec. 16, 2005.

This work was supported by National Institutes of Health Grants MH50314 (S.R.S.), MH29670 (A.A.G.), and MH58921 (R.D.B.) and by a National Alliance for Research on Schizophrenia and Depression Young Investigator Award (L.H.M.).

Correspondence should be addressed to Dr. Susan R. Sesack, Department of Neuroscience, 446 Crawford Hall, University of Pittsburgh, Pittsburgh, PA 15260. E-mail: sesack@bns.pitt.edu.

DOI:10.1523/JNEUROSCI.4450-05.2006

Copyright $\odot 2006$ Society for Neuroscience $\quad$ 0270-6474/06/261571-08\$15.00/0 orders and ADHD, likely via an action within the PFC (Frazer, 2000; Bymaster et al., 2002; Michelson et al., 2003).

The first subcellular characterization of NET in the PFC revealed that $\mathrm{NE}$ axons exhibit unusual characteristics that deviate from traditional models of synthesis and reuptake (Miner et al., 2003). First, NET is localized predominantly within the cytoplasm in the majority of NE axons (85-90\%), an unexpected finding given that NET function depends on its insertion in the plasma membrane. Second, these same profiles do not express detectable levels of tyrosine hydroxylase $(\mathrm{TH})$, raising questions as to how NE can be effectively synthesized.

It is possible that NE terminals obviate the need for $\mathrm{TH}$ by converting extracellular dopamine (DA) to NE in the PFC, as suggested by the scarcity of DA transporters (Sesack et al., 1998), extensive diffusion of DA (Garris and Wightman, 1994; Cass and Gerhardt, 1995), and demonstrated ability of NET to clear DA (Tanda et al., 1994; Gresch et al., 1995; Bymaster et al., 2002). However, one would expect NE axons to express more plasmalemmal NET to facilitate DA uptake. Hence, most NE terminals appear to be in a low activity state, because they can neither synthesize abundant levels of NE precursors nor maximize the synthesis of NE from captured DA. 
In contrast, the minority of NE axons in the PFC (10-15\%) express NET primarily along the plasma membrane and exhibit substantial TH levels (Miner et al., 2003). These axons may function in a high activity state, because they can synthesize NE and recycle both released NE and extracellular DA. These observations imply that NET and TH participate in regulated trafficking events directly linked to NE cell activity, an idea consistent with in vitro studies indicating that cellular activity modulates the surface distribution of NET (Savchenko et al., 2003).

We sought to test this hypothesis in an intact, native system by examining whether the enhanced activity of NE cells induced by chronic stress alters the subcellular distribution of NET and expression of TH in the rat PFC. Chronic stress increases NE neuron responsivity (Simson and Weiss, 1988; Pavcovich and Ramirez, 1991; Conti and Foote, 1996; Mana and Grace, 1997; Jedema et al., 2001) and the levels and activity of TH in the locus ceruleus (LC) (Mamalaki et al., 1992; Watanabe et al., 1995; Rusnak et al., 1998), the source of PFC NE (Loughlin et al., 1982). If NET and TH localization are linked to activity states, then chronic stress should increase plasmalemmal NET and TH expression, thus altering the dynamics of NE transmission and impacting cognition and affect.

\section{Materials and Methods}

Subjects. Naive adult male Sprague Dawley rats (Hilltop Lab Animals, Scottsdale, PA) weighing 300-350 g were used. All procedures were performed in accordance with the National Research Council Guide for the Care and Use of Laboratory Animals (1996), and animal use protocols were approved by the University of Pittsburgh's Institutional Animal Care and Use Committee. To minimize variance attributable to methodological issues, cohorts of randomly assigned rats consisting of two stressed and two control animals (total of four cohorts, 16 rats) were treated and processed together. With the exception of chronic exposure to cold, all of the remaining experimental procedures (e.g., antibody incubations and processing times) were the same between the members of a cohort.

Chronic cold stress paradigm. Rats to be chronically stressed were shaved, because of the more profound effects of chronic cold exposure on adrenal TH after this treatment (Fluharty et al., 1983). Animals were then placed in hanging wire cages in a room maintained at $4^{\circ} \mathrm{C}$ for $14 \mathrm{~d}$. Animals had constant access to food, but the number of pellets was limited to prevent their use as insulation. Animals treated in this manner have been shown to maintain core body temperature (Moore et al., 2001). Water was available ad libitum. Control rats were maintained in the standard animal room for $14 \mathrm{~d}$. The mean body weight of animals was similar at the beginning of treatment (control, $211 \pm 8$ g; stress, $210 \pm$ $12 \mathrm{~g}$ ), whereas control animals gained more weight ( $44 \pm 7 \%)$ than did stressed rats $(33 \pm 5 \%)$ over the subsequent 2 week period (paired $t$ test; $\left.t_{(7)}=2.36 ; p=0.003\right)$.

Immunocytochemistry. The immunocytochemical procedures have been described in detail in our previous study of NET in the PFC (Miner et al., 2003). Anesthetized rats were fixed by intracardial perfusion with $3.75 \%$ acrolein and $2 \%$ paraformaldehyde, and coronal sections through the PFC were cut at $50 \mu \mathrm{m}$ thickness using a vibratome. For ultrastructural investigation, sections were incubated in a mixture of primary antibodies directed against NET and TH with $0.04 \%$ Triton X-100 to enhance penetration and then processed for immunoperoxidase labeling of TH (mouse monoclonal antibody; 1:4000; Chemicon, Temecula, CA) followed by immunogold-silver labeling of NET (rabbit polyclonal antibody; 1:2000). Examination of the subcellular distribution of NET required the nondiffusible immunogold marker, whereas the greater sensitivity of the avidin-biotin immunoperoxidase technique was used to detect potentially low levels of TH (Chan et al., 1990). For light microscopic examination, immunoperoxidase labeling of NET was performed using $0.2 \%$ Triton $\mathrm{X}-100$.

The rabbit polyclonal NET antiserum (Schroeter et al., 2000) was raised against amino acids 585-607 of the $C$ terminus of mouse NET
(Fritz et al., 1998). Specificity has been extensively demonstrated by Western blot analysis, antigen preadsorption, absence of staining after selective NE lesions, light and electron microscopic distribution, dual labeling with dopamine- $\beta$-hydroxylase-immunoreactive ( $\mathrm{D} \beta \mathrm{H}$-ir) profiles (Schroeter et al., 2000; Miner et al., 2003), and absence of labeling in NET knock-out mice (Savchenko et al., 2003). NET is a selective marker for NE cells and processes, because NET mRNA is localized exclusively to $\mathrm{D} \beta \mathrm{H}$-positive neurons (Lorang et al., 1994; Hoffman et al., 1998). Hence, we consider the presence of NET immunoreactivity to be sufficient evidence that labeled profiles in the PFC are NE axons. The mouse monoclonal antibody against rat $\mathrm{TH}$ (Chemicon) recognizes an epitope on the outside of the regulatory $\mathrm{N}$ terminus. This antibody as well as the secondary antisera are commercially available, and the specificity of each has been determined or described in previous publications using techniques such as Western blot analysis and radioactive immunoassay (Steinbusch and Tilders, 1987; Wolf et al., 1991; Sesack et al., 1995).

Light microscopy. Sections for light microscopy were photographed using a Zeiss (Oberkochen, Germany) Axiophot light microscope and a Hamamatsu (Bridgewater, NJ) digital camera. Individual images through the PFC of control and stressed rats were assembled onto a common panel, and then the same adjustments were made for brightness, contrast, and sharpness using Adobe Photoshop (Adobe Systems, San Jose, CA).

Electron microscopy. The sections to be processed for electron microscopy were subjected to osmication, dehydration, and plastic embedding as described previously (Miner et al., 2003). Pieces of the prelimbic PFC (Krettek and Price, 1977) containing either layers I-III or V-VI were cut at $60 \mathrm{~nm}$ using an ultramicrotome (Leica, Buffalo, NY). Ultrathin sections were counterstained with heavy metals and examined using a Zeiss 902 transmission electron microscope.

Criterion for immunolabeling. Immunoperoxidase staining for TH appeared as an electron-dense "flocculent" product over axonal structures. Our criterion for specific immunogold-silver labeling was a minimum of two particles in direct contact with the plasma membrane or three total particles (membraneous and cytoplasmic), the same as originally used to characterize NET (Miner et al., 2003) as well as the serotonin transporter in the PFC (Miner et al., 2000). We acknowledge that our criteria may have resulted in some false negative results for NET. However, the use of the sensitive avidin-biotin immunoperoxidase method to visualize TH minimized false negative outcomes for detection of this protein (Miner et al., 2003).

Tissue sampling and image analysis. A minimum of two vibratome sections each from superficial (I-III) and deep (V-VI) layers from tissue dually labeled for NET and TH were examined for each animal (i.e., a minimum of four sections per animal). Each section was systematically examined until $\sim 25$ NET-ir profiles meeting the criteria above were observed and photographed at $20,000 \times$. Sampling was limited to the surface of the tissue where immunoreagent penetration is maximal. For each photograph, the number of structures showing specific NET immunoreactivity was recorded, and the area and diameter of each profile were determined using a Presage image analysis system (Advanced Imaging Concepts, Princeton, NJ) (Sesack et al., 1998). The following characteristics were also recorded: (1) the number of gold particles per profile, (2) the location of gold particles (membrane vs cytoplasm), (3) the number, type, and target of synaptic contacts being formed, and (4) the presence or absence of TH immunoreactivity.

Statistical analyses. Differences in the average area and diameter of NET-ir profiles were assessed by standard ANOVA ( $\alpha=0.05)$. Differences between overall measures and cortical layer were determined using the nonparametric Fisher's exact test $(\alpha=0.05)$. It should be noted that no differences in the overall proportion of NET associated with the plasma membrane were observed between superficial (I-III) and deep (V-VI) layers in either controls (Fisher's exact test; $p=0.647$ ) or stressed rats (Fisher's exact test; $p=0.395$ ). Likewise, no differences in the expression of TH between superficial and deep layers were revealed in controls (Fisher's exact test; $p=0.999$ ) or stressed rats (Fisher's exact test; $p=0.361)$. Therefore, the reported analyses include data that were collapsed over the layers. The average proportion of NET immunogoldsilver labeling on the membrane was analyzed as a percentage of the 

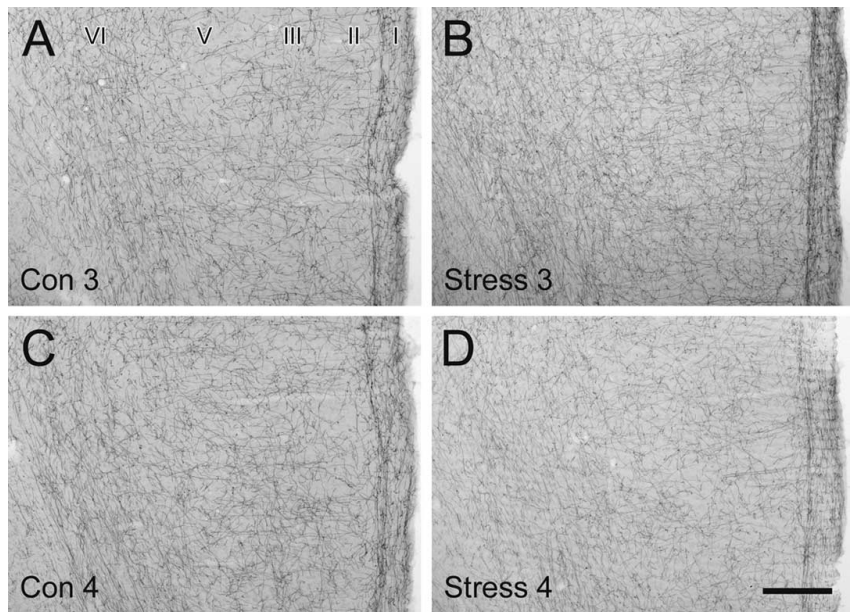

Figure 1. No obvious, consistent qualitative differences in the distribution, density, or general morphology of NET-ir fibers were observed in rats exposed to chronic cold stress. $\boldsymbol{A}-\boldsymbol{D}$, Representative light micrographs showing immunoperoxidase labeling of NET-ir axons in coronal sections through the rat PFC from the second cohort of control (Con) and chronically stressed (Stress) rats (animals 3 and 4). NET-ir fibers exhibit a uniform density across the cortical layers, with an additional dense plexus of axons coursing along the border between layers I and II. Scale bar, $250 \mu \mathrm{m}$.

labeling in the entire profile and as the arc-sine square-root-transformed average percentage. To compare the proportion of gold-silver particles for NET observed on the plasma membrane versus in the cytoplasm, a Poisson regression model was used in which the model effects included cohort, treatment, and their interaction. As expected, no interaction effects were found $\left(\chi^{2}=5.4 ; \mathrm{df}=3 ; p=0.145\right)$; therefore, $p$ values and estimates were based on an additive model. Using this analysis, a significant difference between the cohorts was observed $\left(\chi^{2}=29.89\right.$; $\mathrm{df}=3$; $p<0.001$ ). To analyze the proportion of NE terminals expressing TH immunoreactivity, a repeated-measures logistic regression analysis was used. This model allowed for treatment effect, cohort effect, and treatment-by-cohort interactions. A significant different in the expression of TH between the cohorts was also observed $\left(\chi^{2}=27.54\right.$; $\mathrm{df}=3$; $p<0.001)$; however, no interaction between the stress treatment and the cohorts was found $\left(\chi^{2}=5.23 ; \mathrm{df}=3 ; p=0.156\right)$. Both the Poisson and the logistical regression models accounted for rat variability and permitted multiple dependent observations to be made in the same rat.

\section{Results}

\section{Light microscopic examination of PFC NE axons in control} and chronically stressed rats

NE axons labeled by immunoperoxidase for NET were distributed across all layers of the rat prelimbic PFC in a relatively uniform manner. These relatively smooth NET-ir fibers ran parallel to the pial surface at the layer I-II border and in layer VI while being oriented tangentially in middle layers. No consistent, qualitative differences in the laminar distribution, density, orientation, or morphological characteristics of NET-ir fibers were observed between any of the control and chronically stressed rats. Figure 1 illustrates the similarity in morphology and distribution observed at the light microscopic level for one cohort. Although the labeling for NET in the stress 4 animal (Fig. $1 D$ ) appears to be less intense than the control animals (Fig. $1 A, C$ ), such a difference was not consistently observed in other stressed animals (Fig. $1 B$ ) or in other cohorts. Moreover, these observations did not parallel the electron microscopic findings, which revealed a large (stress 3 ) and a moderate (stress 4) alteration in NET-ir axonal characteristics (see below).

\section{Ultrastructure of NE axons in the PFC of control rats}

In agreement with our previous electron microscopic observations in naive rats (Schroeter et al., 2000; Miner et al., 2003), the majority of NE profiles (87\%) in the prelimbic PFC of control rats exhibited immunogold-silver labeling for NET predominantly within the cytoplasm and no detectable immunoperoxidase staining for $\mathrm{TH}$ (Fig. $2 A, B$ ), despite the sensitivity of the latter method (Hsu et al., 1981). The remaining NE axons (13\%) expressed NET primarily along the plasma membrane and contained prominent TH immunoreactivity (Fig. 2C). In all cases, the labeled profiles exhibited the ultrastructural characteristics of NE terminals (Beaudet and Descarries, 1978, 1984; Seguela et al., 1990; Miner et al., 2003), including lack of myelination, content of small clear, and occasional dense-cored vesicles. As is also characteristic of NE axons, the extent to which NET-ir varicosities were observed to form classical synapses (i.e., the synaptic incidence) was low (130 of 736; 18\%). When observed, synaptic contacts were predominantly of the symmetric type ( 81 of 130 ; $62 \%$ ) and targeted dendritic shafts (88 of $130 ; 68 \%$ ) and spines (42 of $130 ; 32 \%$ ). It is important to note that synaptic contacts were only formed by the axon terminals singly labeled for NET, with NET localized predominantly within the cytoplasm. In those profiles, immunogold-silver labeling for NET was never localized to the perisynaptic region (Fig. $2 B$ ).

\section{Chronic cold stress increases the plasma membrane distribution of NET in NE axons in the rat PFC}

Within the PFC of chronically stressed rats, many NE profiles exhibited an increase in the plasmalemmal distribution of NET (Fig. 3), with the overall percentage increasing significantly from $29 \%$ in control rats to $51 \%$ in chronically stressed rats (Poisson regression; $\chi^{2}=9.1 ; \mathrm{df}=1 ; p=0.003$ ) (Fig. 4, Table 1). Although the extent of this enhanced association of NET with the plasma membrane varied across subjects (Fig. 4), a significant increase was observed on average, and no stressed animals exhibited a reduced plasmalemmal association of NET. In stressed rats, we noted some profiles forming synaptic contacts in which NET immunoreactivity was localized to the immediate perisynaptic region (Fig. 3D). Such a perisynaptic distribution of NET was never observed in control rats.

\section{Chronic cold stress increases the expression of TH in NE axons in the rat PFC}

A greater proportion of NE profiles in rats exposed to chronic stress expressed detectable levels of TH (Figs. 5, 6), increasing from $13 \%$ in control rats to $32 \%$ in stressed animals (logistic regression; $\left.\chi^{2}=61.41 ; \mathrm{df}=1 ; p \leq 0.001\right)$ (Table 1). Although the levels of TH immunoreactivity were greater in all the stressed rats, the magnitude of this increase also varied across subjects (Fig. 6). It should be noted that the intensity of TH labeling in some individual profiles from stressed rats appeared to be qualitatively lower (Fig. 5B) than that typically observed within individual profiles in control and naive animals (Miner et al., 2003). Importantly, we found several examples of terminals dually labeled for NET and TH that formed synaptic contacts and expressed NET immunoreactivity in a perisynaptic location (Fig. $5 C$ ). Such arrangements match traditional expectations of NE terminals but have never been observed in any control or naive animals (present study) (Miner et al., 2003). 


\section{Chronic cold stress does not alter other ultrastructural features of $\mathrm{NE}$ axons in the rat PFC}

It is possible that $\mathrm{NE}$ axons might respond to a persistent increase in activity by altering either the number or size of varicosities or the overall content of NET protein. Hence, it is important to note that the density of NET-ir profiles (i.e., number per $1000 \mu \mathrm{m}^{2}$ of tissue) was not significantly different in chronically stressed compared with control rats (controls, $1.13 \pm 0.52$; stressed, $1.07 \pm 0.48 ; F_{(1,14)}=0.057 ; p=$ $0.815)$ (Table 1). In addition, no differences were observed in the average area or diameter of NET-ir axons: area, $0.37 \pm$ $0.05 \mu \mathrm{m}$ for controls, $0.33 \pm 0.06 \mu \mathrm{m}$ for stressed rats $\left(F_{(1,14)}=3.38 ; p=0.087\right)$ (Table 1); diameter, $0.36 \pm 0.05 \mu \mathrm{m}$ for controls, $0.33 \pm 0.05 \mu \mathrm{m}$ for stressed animals $\left(F_{(1,14)}=2.363 ; p=0.136\right)$. Furthermore, the intensity of NET labeling per profile, as defined by the number of immunogold-silver particles per unit area, varied substantially across axons in both control (24.25 \pm 30.04) and stressed $(27.74 \pm 50.96)$ rats but yielded no significant differences between the groups $\left(F_{(1,14)}=3.38 ; p=0.087\right)$ (Table 1). Finally, the synaptic incidence for NET-ir terminals did not differ (Fisher's exact test; $p=0.563)$ between control (18\%) and stressed $(103$ of $742 ; 14 \%)$ rats; there was also no difference in the types of synaptic contacts, being primarily of the symmetric type ( 80 of $103 ; 77 \%$ ) onto dendritic shafts (64 of 103; 62\%) in stressed animals.

\section{Discussion}

These findings constitute the first demonstration of activity-dependent trafficking of NET and expression of TH in an intact neuronal system while providing provocative information about the response of the NE system to chronic stress. The results support our hypothesis that the plasmalemmal association of NET and detectability of TH in the PFC are dynamically regulated in response to chronic alterations in the activity of NE neurons (supplemental Fig. S1, available at www.jneurosci.org as supplemental material). These data imply that in naive/control rats, the majority of NE terminals that contain predominantly cytoplasmic NET and lack detectable TH reflect a low activity state for this system. However, the elevation of plasmalemmal NET and TH expression after chronic cold stress suggests that these terminals alter their characteristics in response to increased drive. The alteration in NET distribution in particular provides a means for terminals to rapidly switch from a low activity "idling" state to one of highly efficient NE recycling in response to sustained behavioral demand.
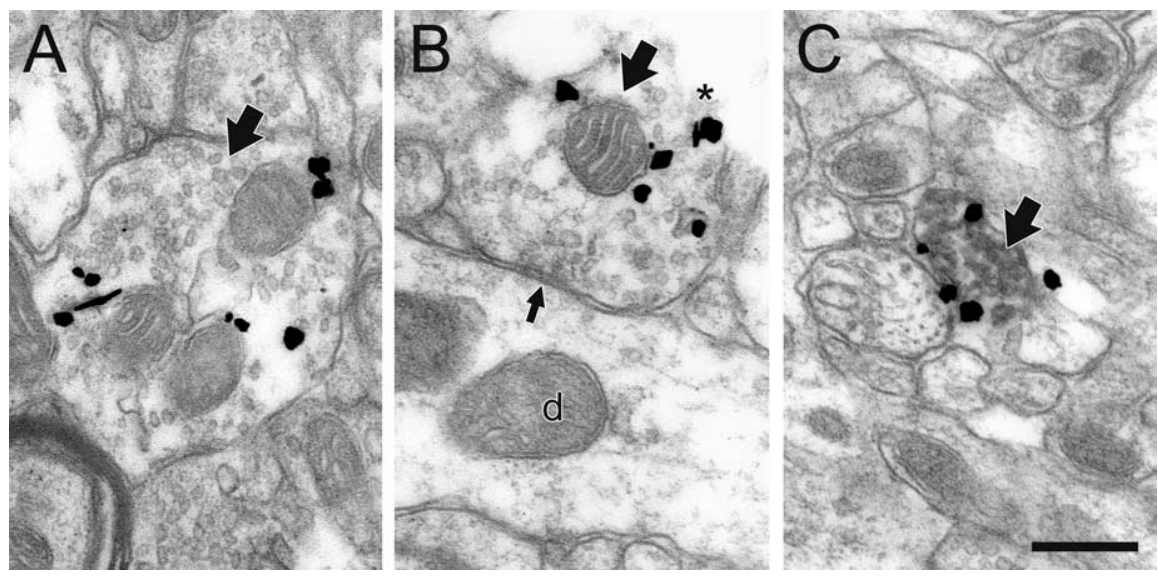

Figure 2. NE axons and terminals (large black arrows) exhibit unusual immunoreactive characteristics in the PFC of control rats. $A, B$, By electron microscopic examination, most profiles express gold-silver labeling for NET predominantly in the cytoplasm and contain no detectable immunoperoxidase product for $\mathrm{TH}$, even when they are cut open at the tissue surface where antibody penetration is maximal (asterisk in $\boldsymbol{B}$ ). These profiles only occasionally form synapses, most commonly symmetric contacts (small black arrow in $\boldsymbol{B}$ ) onto dendrites (d), and NET is not localized near these synapses. $\boldsymbol{C}$, Only a minority of NE axons express NET predominantly on the plasma membrane, and these profiles also exhibit peroxidase labeling for TH. Scale bar, $0.48 \mu \mathrm{m}$.
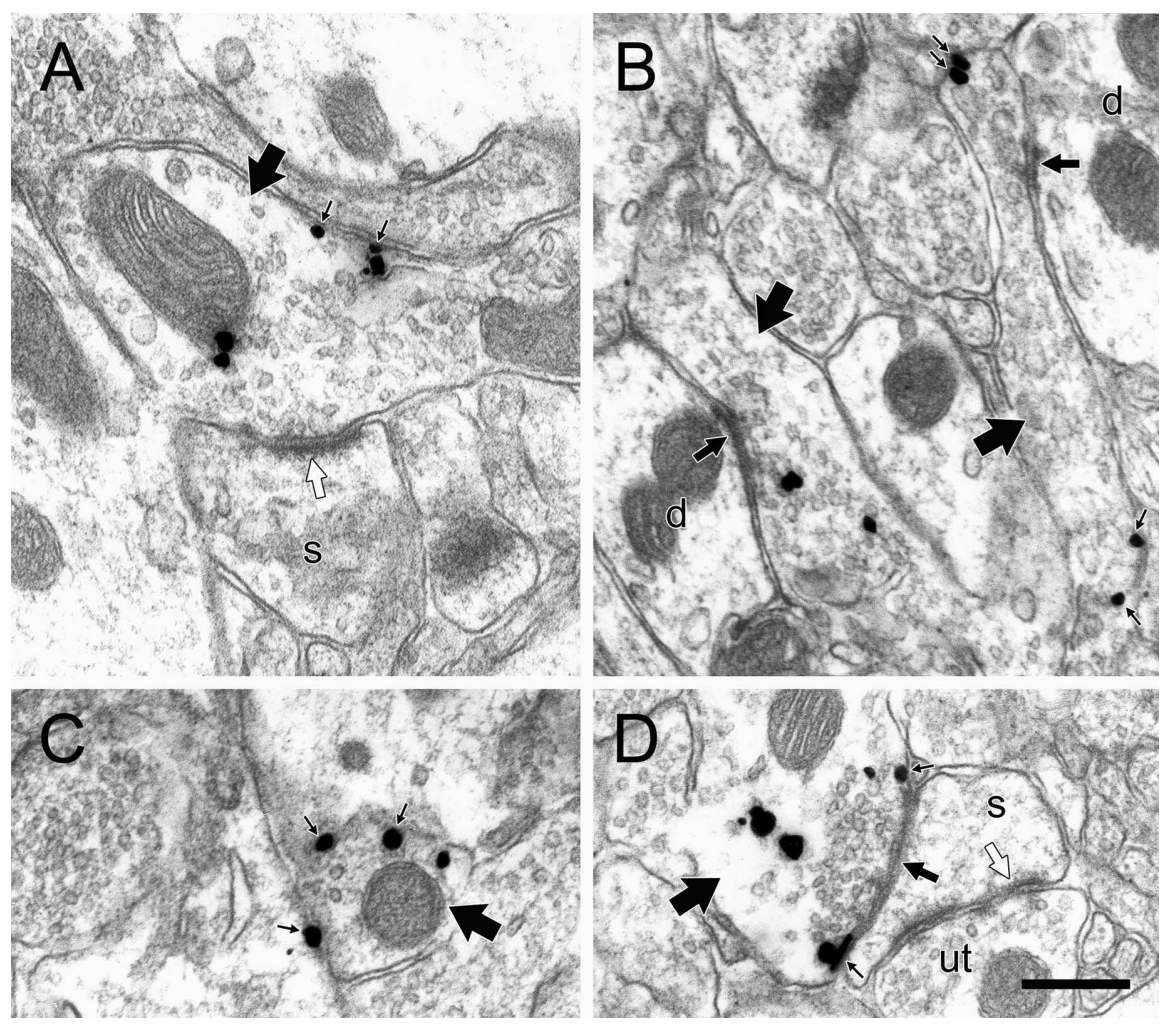

Figure 3. NE profiles in the PFC of chronically stressed rats exhibit immunolabeling characteristics not typically observed in control animals. $\boldsymbol{A}-\boldsymbol{D}$, Electron micrographs of axons and varicosities singly labeled for NET (large arrows) in chronically stressed rats exhibit immunogold-silver labeling more frequently along the plasma membrane (small black arrows), even in profiles forming symmetric (medium black arrows) or asymmetric (white arrows) synapses onto unlabeled dendrites (d) or spines (s). Occasionally, NET is localized to the immediate perisynaptic region (D), an event never observed in control rats. The dendritic spine in $\boldsymbol{D}$ also receives synaptic input (white arrow) from an unlabeled terminal (ut). Despite the increase in plasmalemmal NET expression, none of the profiles shown here exhibit peroxidase reaction product for TH. Scale bar, $0.48 \mu \mathrm{m}$.

The observed changes in NET and TH likely reflect the chronic aversive nature of continuous exposure to cold rather than the physiological response to cold itself. More specifically, adaptive thermoregulation is expressed by at least 1 week of cold exposure and probably earlier, given the healthy condition of the 


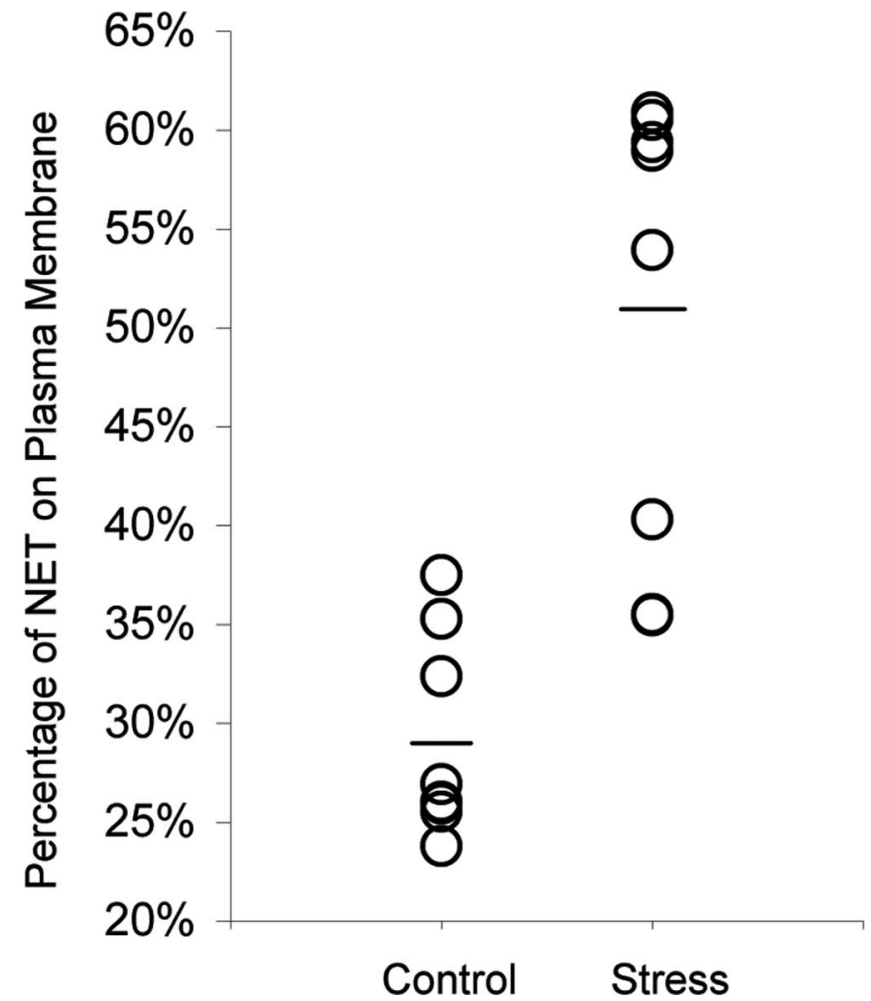

Figure 4. The extent to which chronic cold stress increases the proportion of NET associated with the plasma membrane varies across individual subjects. The mean proportion of plasmalemmal NET for each group is indicated by a horizontal bar.

Table 1. NET-immunoreactive profiles in the prefrontal cortex of control and chronically stressed rats

\begin{tabular}{lll}
\hline & Control & Stress \\
\hline Number of subjects & 16 & 16 \\
Area of tissue examined $\left(\mu \mathrm{m}^{2}\right)$ & 763,902 & 795,275 \\
$\begin{array}{l}\text { Number of NET-ir profiles per 1000 } \mu \mathrm{m}^{2} \text { of tissue } \\
\text { Mean area of NET-ir profiles }\left(\mu \mathrm{m}^{2}\right)\end{array}$ & 1.13 & 1.07 \\
$\begin{array}{l}\text { Mean density of gold labeling for NET (particles } \\
\quad \text { per unit area) }\end{array}$ & 0.37 & 0.33 \\
$\begin{array}{l}\text { Percentage of gold labeling for NET on the plasma } \\
\quad \text { membrane }\end{array}$ & 24.2 & 27.7 \\
$\begin{array}{l}\text { Percentage of NET-ir profiles dually labeled for TH } \\
\text { Percentage of NET-ir profiles forming synapses in }\end{array}$ & $29 \%$ & $51 \%^{*}$ \\
$\quad$ single sections & $13 \%$ & $32 \%^{* *}$ \\
\end{tabular}

*Poisson regression; $\chi^{2}=9.1 ; \mathrm{df}=1 ; p=0.003$.

**ogistical regression; $\chi^{2}=61.41 ; \mathrm{df}=1 ; p \leq 0.001$.

All other comparisons, not significant.

animals (Moore et al., 2001). Conversely, the PFC NE system shows increased responsivity only after 2 weeks and not 1 week of chronic cold (Finlay et al., 1997). The different time courses of these processes suggest independent mechanisms.

The present population study provides no direct evidence for alterations in NET and TH within individual NE terminals. However, the lack of systematic changes in the overall density of NE axons suggests that the enhancement of plasmalemmal NET and detectable TH are unlikely to reflect either sprouting or degeneration of NE axons, as reported after long-term stress (Sakaguchi and Nakamura, 1990; Kitayama et al., 1997). Hence, our results are most consistent with a model in which individual NE axons undergo alterations in NET trafficking and $\mathrm{TH}$ expression in response to the heightened activity demand produced by chronic stress.

The present findings provide a crucial component in understanding the overall response of the PFC NE system to chronic stress. Previous studies have established that the excitability of LC neurons is increased after chronic cold stress (Mana and Grace, 1997; Jedema et al., 2001; Jedema and Grace, 2003). However, contrary to what would be expected based on this enhancement, basal extracellular levels of NE in the PFC are not significantly higher after this treatment (Gresch et al., 1994; Jedema et al., 1999). As shown here, this discrepancy may at least partly reflect an augmentation in the plasma membrane location of NET, which would counteract an increase in NE release. These findings strengthen the idea that local influences participate in the NE response to chronic stress within the PFC.

The current findings supply the first demonstration of increases in TH protein levels in NE terminals after chronic cold stress. Despite clear evidence for increases in TH activity, mRNA, and protein in the LC evoked by chronic stress (Mamalaki et al., 1992; Watanabe et al., 1995; Rusnak et al., 1998), no increase in basal TH activity in terminal regions has been reported (Nisenbaum et al., 1991). Although not yet examined directly in the PFC, such changes in TH activity or levels might not be observed, because the majority of TH in this region ( $90 \%)$ is expressed in DA axons (Schmidt and Bhatnagar, 1979; Miner et al., 2003). This situation would effectively mask a selective increase in $\mathrm{TH}$ activity in NE terminals. Hence, our method provides the advantage of selectively detecting increases in TH expression within axon varicosities specifically identified to be NE.

Our findings also offer the first functional insight into the basis for the absence of detectable $\mathrm{TH}$ from many cortical NE terminals, as shown in light microscopic and supportive biochemical studies of rats and monkeys over the past three decades (Pickel et al., 1975a; Schmidt and Bhatnagar, 1979; Lewis et al., 1987; Noack and Lewis, 1989). These observations have always been difficult to understand, given that $\mathrm{TH}$ is readily detected in LC cell bodies (Pickel et al., 1975b) and that catecholamine synthesis requires TH. The higher levels of TH expression in PFC NE axons observed after exposure to chronic stress suggest that this enzyme can upregulate in response to a heightened demand for synthesis. In naive/control rats, TH labeling in the minority population of NE axons appears as "all or none," in that when it is present, it is typically intense. The more graded levels of $\mathrm{TH}$ observed in stressed rats in the present study [compare Fig. $6 B$ with Miner et al. (2003), their Fig. 5A] may be attributable to the mobilization of $\mathrm{TH}$ into terminals that did not formerly express the enzyme.

It is important to note that NET provides the main mechanism for clearing extracellular DA in the PFC (Tanda et al., 1994; Gresch et al., 1995; Yamamoto and Novotney, 1998; Bymaster et al., 2002). Hence, under low activity conditions, NE axons may not require high levels of $\mathrm{TH}$, because they can use extracellular DA as a precursor for NE. The low levels of plasmalemmal NET observed in the PFC of naive rats are somewhat inconsistent with this model (Miner et al., 2003). However, the hypothesis is supported by the observation that NE axons more often express detectable TH in cortical areas that receive sparse DA afferents (Noack and Lewis, 1989). In any case, the fact that chronic stress augments plasmalemmal NET in some axons that do not show concomitantly increased TH expression suggests that these fibers may initially attempt to maximize their uptake of DA to meet the increased demand for transmitter.

The less than maximal alterations in NET and TH induced by 
cold exposure suggest that additional factors may operate to counteract the impact of chronic stress. It is possible that enhancing the severity of the stressor (i.e., duration and/or intensity) might lead to greater changes in NET and TH. A second possibility is that the constant and predictable application of this physiological stressor may have allowed the NE system a certain degree of adaptation. This could be tested by using a paradigm involving an intermittent, unpredictable stressor. However, this type of stress has not been shown to produce as marked a change in neurochemical measures of PFC NE transmission (Jedema et al., 1999). Finally, it is possible that the availability of extracellular DA in the PFC continues to assist NE terminals in maintaining normal transmitter levels even in heightened activity states. This is supported by the observation of a trend for decreased basal extracellular DA levels in
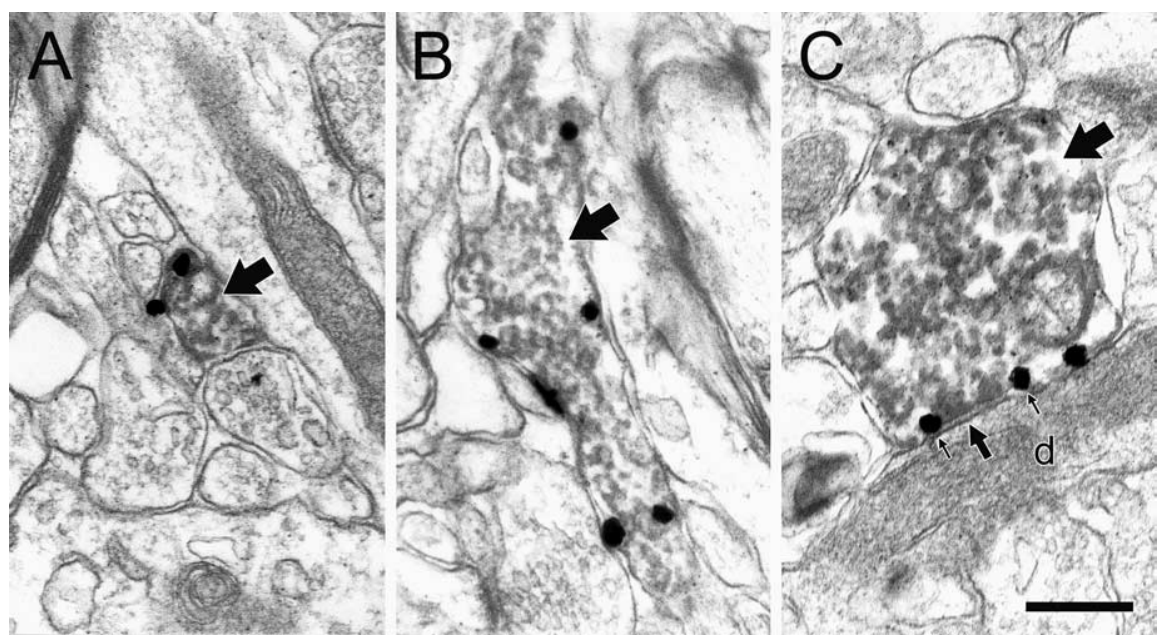

Figure 5. $A-C$, Chronic cold stress increases the proportion of NE axons dually labeled for NET and TH (large arrows). Electron microscopic images illustrate that immunogold-silver particles for NET are predominantly associated with the plasma membrane in these profiles. In C, the dually labeled profile forms a symmetric synapse (medium black arrow) onto a dendritic shaft (d), and gold-silver particles (small black arrows) are localized to the plasma membrane immediately surrounding the synaptic contact. Neither of these two observations was ever made in control rats. Scale bar, $0.48 \mu \mathrm{m}$. the PFC in response to chronic cold stress (Gresch et al., 1994; Finlay et al., 1995), implying that more DA is being cleared by NET for use in NE synthesis.

Although the direction of NET and TH changes evoked by chronic stress was similar across all rat pairs, the magnitude of change between individual subjects was variable. Such observations are consistent with individual variation in chronic stressinduced NE transmission in other terminal regions that correlate with behavioral responsivity (Rosario and Abercrombie, 1999). In this regard, individual differences such as genetic predisposition have been suggested as factors in determining one's vulnerability to stress and susceptibility to depression or anxiety disorders (Kendler et al., 1995). Hence, the extent of plasmalemmal NET and TH expression in the PFC of rats would be predicted to correlate with differences in their genetic susceptibility to stress (Tejani-Butt et al., 1994; Rex et al., 1996; Pardon et al., 2002). In line with this, Hahn et al. (2005) reported recently that naturally occurring genetic variations in human NET can limit the ability of the transporter to respond to regulatory stimuli. We suggest that in subjects carrying such variants, homeostatic responses such as those noted here may be limited in a manner that impacts risk for cognitive or affective disorders.

Chronic stress can precipitate depressive symptoms in humans (Hammen, 1991), and prolonged exposure of rats to various stressors results in many of the motivational, neuroendocrine, anhedonic, and behavioral characteristics observed in human depression (Brady, 1994). Hence, chronic exposure to a physiologically relevant stressor has been proposed as an animal model of depression (Gambarana et al., 2001). If so, then the stress-evoked alterations in PFC NET and TH observed here are likely to be involved in the pathophysiology of affective disorders.

For the treatment of depression, selective NE reuptake inhibitors (SNRIs) are effective therapeutic agents after several weeks of administration (Frazer, 2000). In general, long-term treatment with SNRIs decreases LC activity, TH expression, and NET function in a manner opposite to that produced by chronic stress exposure (Komori et al., 1992; Grant and Weiss, 2001; Szabo and Blier, 2001; Benmansour et al., 2004). Moreover, long-term antidepressant treatment attenuates many of the changes induced by chronic stress, suggesting that such actions contribute to their

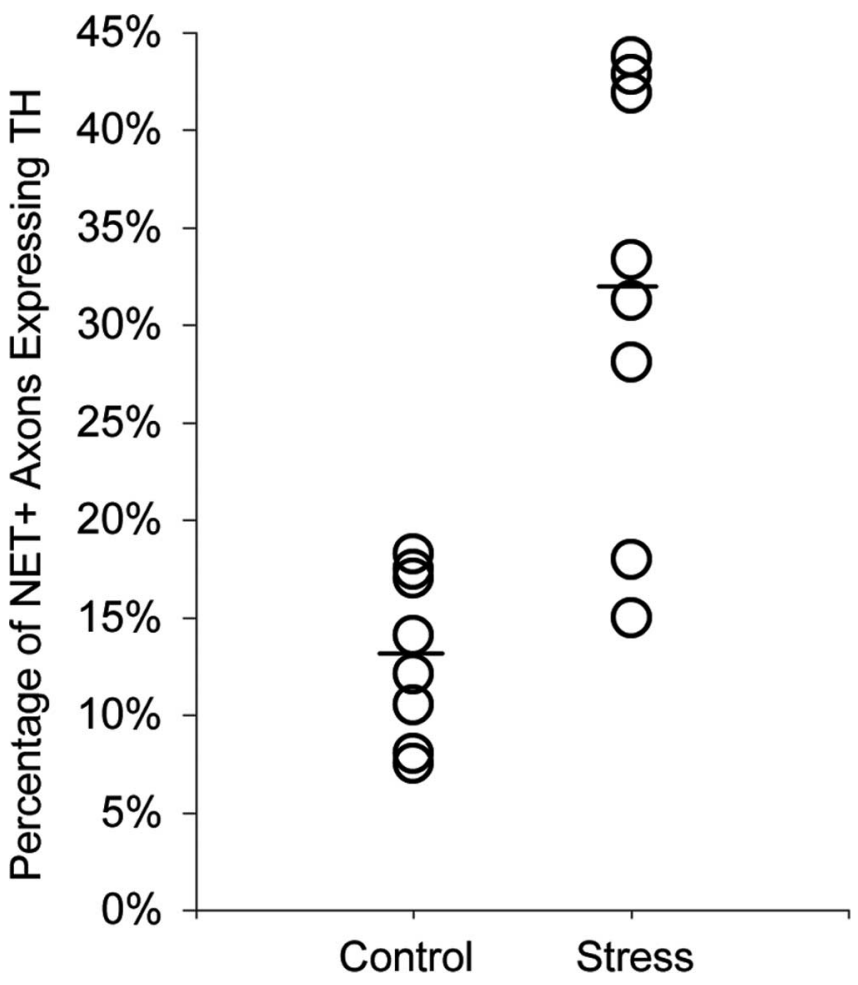

Figure 6. The extent to which chronic cold stress increases the proportion of NET-ir axons that also express TH varies across individual subjects. The mean proportion for each group is indicated by a horizontal bar.

therapeutic efficacy (Melia et al., 1992a,b). The findings of the present study suggest that alterations in NET location may be involved in the etiology of affective disorders. It remains to be determined whether the therapeutic properties of SNRIs may be at least partially mediated by alterations in the plasma membrane distribution of this transporter.

\section{References}

Aston-Jones G, Fajkowski J, Cohen J (1999) Role of the locus coeruleus in attention and behavioral flexibility. Biol Psychiatry 46:1309-1320. 
Beaudet A, Descarries L (1978) The monoamine innervation of rat cerebral cortex: synaptic and nonsynaptic axon terminals. Neuroscience 3:851-860.

Beaudet A, Descarries L (1984) Fine structure of monoamine axon terminals in cerebral cortex. In: Monoamine innervation of the cerebral cortex (Descarries L, Reader T, Jasper H, eds), pp 77-93. New York: Liss.

Benmansour S, Altamirano AV, Jones DJ, Sanchez TA, Gould GG, Pardon M-C, Morilak DA, Frazer A (2004) Regulation of the norepinephrine transporter by chronic administration of antidepressants. Biol Psychiatry 55:313-316.

Biederman J, Spencer T (1999) Attention-deficit/hyperactivity disorder (ADHD) as a noradrenergic disorder. Biol Psychiatry 46:1234-1242.

Blakely RD, Bauman AL (2000) Biogenic amine transporters: regulation in flux. Curr Opin Neurobiol 10:328-336.

Brady LS (1994) Stress, antidepressant drugs, and the locus coeruleus. Brain Res Bull 35:545-556.

Bymaster FP, Katner JS, Nelson DL, Hemrick-Luecke SK, Threlkeld PG, Heiligenstein JH, Morin SM, Gehlert DR, Perry KW (2002) Atomoxetine increases extracellular levels of norepinephrine and dopamine in the prefrontal cortex of the rat: a potential mechanism for efficacy in attention deficit / hyperactivity disorder. Neuropsychopharmacology 27:699-711.

Callado LF, Meana JJ, Grijalba B (1998) Selective increase of alpha2adrenoceptor agonist binding sites in brains of depressed suicide victims. J Neurochem 70:1114-1123.

Cass WA, Gerhardt GA (1995) In vivo assessment of dopamine uptake in rat medial prefrontal cortex: comparison with dorsal striatum and nucleus accumbens. J Neurochem 65:201-207.

Chan J, Aoki C, Pickel VM (1990) Optimization of differential immunogold-silver and peroxidase labeling with maintenance of ultrastructure in brain sections before plastic embedding. J Neurosci Methods 33:113-127.

Conti LH, Foote SL (1996) Reciprocal cross-desensitization of locus coeruleus electrophysiological responsivity to corticotrophin-releasing factor and stress. Brain Res 722:19-29.

Finlay JM, Zigmond MJ, Abercrombie ED (1995) Increased dopamine and norepinephrine release in medial prefrontal cortex induced by acute and chronic stress: effects of diazepam. Neuroscience 64:619-628.

Finlay JM, Jedema HP, Rabinovic AD, Mana MJ, Zigmond MJ, Sved AF (1997) Impact of corticotropin-releasing hormone on extracellular norepinephrine in prefrontal cortex after chronic cod stress. J Neurochem 69:144-150.

Fluharty SJ, Snyder GL, Stricker EM, Zigmond MJ (1983) Short- and longterm changes in adrenal tyrosine hydroxylase activity during insulininduced hypoglycemia and cold stress. Brain Res 267:384-387.

Frazer A (2000) Norepinephrine involvement in antidepressant action. J Clin Psychiatry 61 [Suppl 10]:25-30.

Fritz JD, Jayanthi LD, Thoreson MA, Blakely RD (1998) Cloning and chromosomal mapping of the murine norepinephrine transporter. J Neurochem 70:2241-2251.

Gambarana C, Scheggi S, Tagliamonte A, Tolu P, DeMontis MG (2001) Animal models for the study of antidepressant activity. Brain Res Brain Res Protoc 7:11-20.

Garris PA, Wightman RM (1994) Different kinetics govern dopaminergic transmission in the amygdala, prefrontal cortex, and striatum: an in vivo voltametric study. J Neurosci 14:442-450.

Grant MM, Weiss JM (2001) Effects of chronic antidepressant drug administration and electroconvulsive shock on locus coeruleus electrophysiological activity. Biol Psychiatry 49:117-129.

Gresch PJ, Sved AF, Zigmond MJ, Finlay JM (1994) Stress-induced sensitization of dopamine and norepinephrine efflux in medial prefrontal cortex of the rat. J Neurochem 63:575-583.

Gresch PJ, Sved AF, Zigmond MJ, Finlay JM (1995) Local influences of endogenous norepinephrine on extracellular dopamine in rat medial prefrontal cortex. J Neurochem 65:111-116.

Hahn MK, Mazei-Robison MS, Blakely RD (2005) Single nucleotide polymorphisms in the human norepinephrine transporter gene affect expression, trafficking, antidepressant interaction, and protein kinase $C$ regulation. Mol Pharmacol 68:457-466.

Hammen C (1991) Generation of stress in the course of unipolar depression. J Abnorm Psychol 100:555-561.

Hoffman BJ, Hansson SR, Mezey E, Palkovits M (1998) Localization and dynamic regulation of biogenic amine transporters in the mammalian central nervous system. Front Neuroendocrinol 19:187-231.

Hsu S-M, Raine L, Fanger H (1981) Use of avidin-biotin-peroxidase complex $(\mathrm{ABC})$ in immunoperoxidase techniques. J Histochem Cytochem 29:577-580.

Jedema HP, Grace AA (2003) Chronic exposure to cold stress alters electrophysiological properties of locus coeruleus neurons recorded in vitro. Neuropsychopharmacology 28:63-72.

Jedema HP, Sved AF, Zigmond MJ, Finlay JM (1999) Sensitization of norepinephrine release in medial prefrontal cortex: effect of different chronic stress protocols. Brain Res 830:211-217.

Jedema HP, Finlay JM, Sved AF, Grace AA (2001) Chronic cold exposure potentiates $\mathrm{CRH}$-evoked increases in electrophysiologic activity of locus coeruleus neurons. Biol Psychiatry 49:351-359.

Kendler KS, Kessler RC, Walters EE, MacLean C, Neale MC, Heath AC, Eaves LJ (1995) Stressful life events, genetic liability, and onset of an episode of major depression in women. Am J Psychiatry 152:833-842.

Kitayama I, Yaga T, Kayahara T, Nakano K, Murase S, Otani M, Nomura J (1997) Long-term stress degenerates, but imipramine generates, noradrenergic axons in the rat cerebral cortex. Biol Psychiatry 42:687-696.

Komori K, Kunimi Y, Yamaoka K, Ito T, Kasahara Y, Nagatsu I (1992) Semiquantitative analysis of immunoreactivities of tyrosine hydroxylase and aromatic L-amino acid decarboxylase in the locus coeruleus of desipramine-treated rats. Neurosci Lett 147:197-200.

Krettek JE, Price JL (1977) The cortical projections of the mediodorsal nucleus and adjacent thalamic nuclei in the rat. J Comp Neurol 171:157-192.

Lewis DA, Campbell MJ, Foote SL, Goldstein M, Morrison JH (1987) The distribution of tyrosine hydroxylase-immunoreactive fibers in primate neocortex is widespread but regionally specific. J Neurosci 7:279-290.

Lorang D, Amara SG, Simerly RB (1994) Cell-type-specific expression of catecholamine transporters in the rat brain. J Neurosci 14:4903-4914.

Loughlin SE, Foote SL, Fallon JH (1982) Locus coeruleus projections to the cortex: topography, morphology and collaterization. Brain Res Bull 9:287-294.

Mamalaki E, Kvetnansky R, Brady LS, Gold PW, Herkenham M (1992) Repeated immobilization stress alters tyrosine hydroxylase, corticotropinreleasing hormone and corticosteroid receptor messenger ribonucleic acid levels in rat brain. J Neuroendocrinol 4:689-699.

Mana MJ, Grace AA (1997) Chronic cold stress alters basal and evoked electrophysiological activity of rat locus coeruleus neurons. Neuroscience 81:1055-1064.

Melia KR, Nestler EJ, Duman RS (1992a) Chronic imipramine treatment normalizes levels of tyrosine hydroxylase in the locus coeruleus of chronically stress rats. Psychopharmacology 108:23-26.

Melia KR, Rasmussen K, Terwilliger RZ, Haycock JW, Nestler EJ, Duman RS (1992b) Coordinate regulation of the cyclic AMP system with firing rate and expression of tyrosine hydroxylase in the rat locus coeruleus: effects of chronic stress and drug treatment. J Neurochem 58:494-502.

Michelson D, Adler L, Spencer T, Reimherr FW, West SA, Allen AJ, Kelsey D, Wernicke J, Dietrich A, Milton D (2003) Atomoxetine in adults with ADHD: two randomized, placebo-controlled studies. Biol Psychiatry 53:112-120.

Miner LAH, Schroeter S, Blakely RD, Sesack SR (2000) Ultrastructural localization of the serotonin transporter in superficial and deep layers of the rat prelimbic prefrontal cortex and its spatial relationship to dopamine terminals. J Comp Neurol 427:220-234.

Miner LAH, Schroeter S, Blakely RD, Sesack SR (2003) Ultrastructural localization of the norepinephrine transporter in superficial and deep layers of the rat prelimbic prefrontal cortex and its spatial relationship to probable dopamine terminals. J Comp Neurol 466:478-494.

Moore H, Rose HJ, Grace AA (2001) Chronic cold stress reduces the spontaneous activity of ventral tegmental dopamine neurons. Neuropsychopharmacology 24:410-419.

Nisenbaum LK, Zigmond MJ, Sved AF, Abercrombie ED (1991) Prior exposure to chronic stress results in enhanced synthesis and release of hippocampal norepinephrine in response to a novel stressor. J Neurosci 11:1478-1484.

Noack HJ, Lewis DA (1989) Antibodies directed against tyrosine hydroxylase differentially recognize noradrenergic axons in monkey neocortex. Brain Res 500:313-324.

Pardon M-C, Gould GG, Garcia A, Phillips L, Cook MC, Miller SA, Mason PA, Morilak DA (2002) Stress reactivity of the brain noradrenergic sys- 
tem in three rat strains differing in their neuroendocrine and behavioral responses to stress: implications for susceptibility to stress-related neuropsychiatric disorders. Neuroscience 115:229-242.

Pavcovich LA, Ramirez OA (1991) Time course of uncontrollable stress in locus coeruleus neuronal activity. Brain Res Bull 26:17-21.

Pickel VM, Joh TH, Field PM, Becker CG, Reis DJ (1975a) Cellular localization of tyrosine hydroxylase by immunocytochemistry. J Histochem Cytochem 23:1-12.

Pickel VM, Joh TH, Reis DJ (1975b) Ultrastructural localization of tyrosine hydroxylase in noradrenergic neurons of brain. Proc Natl Acad Sci USA 72:659-663.

Rex A, Sondern U, Voight JP, Franck S, Fink H (1996) Strain differences in fear-motivated behavior of rats. Pharmacol Biochem Behav 54:107-111.

Robbins TW (1984) Cortical noradrenaline, attention and arousal. Psychol Med 14:13-21.

Rosario LA, Abercrombie ED (1999) Individual differences in behavioral reactivity: correlation with stress-induced norepinephrine efflus in the hippocampus of Sprague-Dawley rats. Brain Res Bull 48:595-602.

Rusnak M, Zorad S, Buckendahl P, Sabban EL, Kvetnansky R (1998) Tyrosine hydroxylase mRNA levels in locus coeruleus of rats during adaptation to long-term immobilization stress exposure. Mol Chem Neuropathol 33:249-258.

Sakaguchi T, Nakamura T (1990) Duration-dependent effects of repeated restraint stress on cortical projections of locus coeruleus neurons. Neurosci Lett 118:193-196.

Savchenko V, Sung U, Blakely RD (2003) Cell surface trafficking of the antidepressant-sensitive norepinephrine transporter revealed with an ectodomain antibody. Mol Cell Neurosci 24:1131-1150.

Schmidt RH, Bhatnagar RK (1979) Assessment of the effects of neonatal subcutaneous 6-hydroxydopamine on noradrenergic and dopaminergic innervation of the cerebral cortex. Brain Res 166:309-319.

Schroeter S, Apparsundaram S, Wiley RG, Miner LAH, Sesack SR, Blakely RD (2000) Immunolocalization of the cocaine- and antidepressant-sensitive 1-norepinephrine transporter. J Comp Neurol 420:211-232.

Seguela P, Watkins KC, Geffard M, Descarries L (1990) Noradrenaline axon terminals in adult rat neocortex: an immunocytochemical analysis in serial thin sections. Neuroscience 35:249-264.

Sesack SR, Bressler CN, Lewis DA (1995) Ultrastructural associations between dopamine terminals and local circuit neurons in the monkey pre- frontal cortex: a study of calretinin-immunoreactive cells. Neurosci Lett 200:9-12.

Sesack SR, Hawrylak VA, Matus C, Guido MA, Levey AI (1998) Dopamine axon varicosities in the prelimbic division of the rat prefrontal cortex exhibit sparse immunoreactivity for the dopamine transporter. J Neurosci 18:2697-2708.

Simson PE, Weiss JM (1988) Altered activity of the locus coeruleus in an animal model of depression. Neuropsychopharmacology 1:287-294.

Southwick SM, Bremner JD, Rasmusson A, Morgan DA, Arnsten AFT, Charney DS (1999) Role of norepinephrine in the pathophysiology and treatment of posttraumatic stress disorder. Biol Psychiatry 46:1192-1204.

Stanford SC (1995) Central noradrenergic neurons and stress. Pharmacol Ther 68:297-342.

Steinbusch HWM, Tilders FJH (1987) Immunohistochemical techniques for light-microscopical localization of dopamine, noradrenaline, serotonin and histamine in the central nervous system. In: Monoaminergic neurons: light microscopy and ultrastructure (Steinbusch H, ed), pp 125166. New York: Wiley.

Szabo ST, Blier P (2001) Effect of the selective noradrenergic reuptake inhibitor reboxetine on the firing activity of noradrenaline and serotonin neurons. Eur J Neurosci 13:2077-2087.

Tanda GL, Carboni E, Frau R, DiChiara G (1994) Increase of extracellular dopamine in the prefrontal cortex: portrait of drugs with antidepressant potential? Psychopharmacology 115:285-288.

Tejani-Butt SM, Pare WP, Yang J (1994) Effect of repeated novel stressors on depressive behavior and brain norepinephrine receptor system in Sprague-Dawley and Wistar Kyoto (WKY) rats. Brain Res 649:27-35.

Watanabe Y, McKittrick CR, Blanchard DC, Blanchard RJ, McEwen BS, Sakai RR (1995) Effects of chronic social stress on tyrosine hydroxylase mRNA and protein levels. Brain Res Mol Brain Res 32:176-180.

Wolf ME, LeWitt PA, Bannon MJ, Dragovic LJ, Kapatos G (1991) Effect of aging on tyrosine hydroxylase protein and the relative number of dopamine nerve terminals in human caudate. J Neurochem 56:1191-1200.

Yamamoto BK, NovotneyS (1998) Regulation of extracellular dopamine by the norepinephrine transporter. J Neurochem 71:274-280.

Zigmond MJ, Finlay JM, Sved AF (1995) Neurochemical studies of central noradrenergic responses to acute and chronic stress. In: Neurobiological and clinical consequences of stress: from normal adaptations to PTSD (Friedman M, Charney D, Deutsch A, eds), pp 45-60. Philadelphia: Lippencott-Raven. 